\title{
Superluminal neutrinos from Lorentz-violating dimension-5 operators
}

\author{
C.A.G. Almeida ${ }^{2}$, M.A. Anacleto ${ }^{1}$, F.A. Brito ${ }^{1, \mathrm{a}}$, E. Passos $^{1}$ \\ ${ }^{1}$ Departamento de Física, Universidade Federal de Campina Grande, Caixa Postal 10071, 58109-970 Campina Grande, Paraíba, Brazil \\ ${ }^{2}$ Departamento de Ciências Exatas, Universidade Federal da Paraíba, 58297-000 Rio Tinto, Paraíba, Brazil
}

Received: 10 November 2011 / Revised: 13 December 2011 / Published online: 3 January 2012

(C) The Author(s) 2011. This article is published with open access at Springerlink.com

\begin{abstract}
We consider Lorentz- and CPT-violating dimension-5 operators to address the issue of superluminal neutrinos recently pointed out in OPERA experiments. We assume these operators in the photon and neutrino sectors to be coupled to Lorentz-violating backgrounds in a preferred frame defined by a time-like direction. We show that such operators can produce a curve with OPERA's slope that fits OPERA, MINOS and supernova SN1987a data.
\end{abstract}

In this letter we consider Extended Myers-Pospelov dimension-5 operators in order to consider physics in a preferred frame with time-like direction in the presence of a Lorentzviolating background $n_{\mu}$ to address the issue of the superluminal neutrinos detected in the OPERA's experiments very recently [1] - for some recent theoretical developments see, for example [2-26]. We derive the dispersion relations associated to the effective Lagrangian for Dirac and Maxwell terms supplemented by dimension- 5 operators given in the form

$$
\begin{aligned}
\mathcal{L}_{\text {eff }}= & \bar{\psi}(i \not \partial-m) \psi+\tilde{g} \bar{\psi} \hbar h \gamma_{5} \hat{D} \psi-\frac{1}{4} F_{\mu \nu} F^{\mu \nu} \\
& +g \epsilon^{\alpha \mu \lambda \rho} n_{\lambda} \hat{D} F_{\rho \alpha} A_{\mu}+\cdots
\end{aligned}
$$

where $(\cdots)$ means interacting terms, $\tilde{g}=\eta / M$ and $g=$ $\xi / M$ with $\eta, \xi$ dimensionless parameters. The dimension-5 operator in the electromagnetic sector is CPT-odd and even under charge conjugation, whereas the dimension-5 operator in the fermionic sector breaks CPT and is even under charge conjugation, $M$ is the mass where new physics such as Lorentz and CPT symmetry violation emerges and $\hat{D}$ is a derivative operator given by

$\hat{D}=(\partial \cdot n)^{2}-\partial^{2} n^{2}$.

a e-mail: fabrito@df.ufcg.edu.br
This is the Myers-Pospelov operator [27] suitably extended in the present study to affect the superluminality behavior at large momenta. Note this reduces to the original operator for light-like backgrounds, i.e., for $n^{2}=0$. The fermionic sector with $\tilde{g} \hat{D}_{\not h} \gamma_{5} \rightarrow-\not b \gamma_{5}$ reduces to CPT-odd extended QED by Colladay and Kostelecky [28], which has been recently considered in superluminal neutrino issues [29]. Similarly, the electromagnetic sector with $g n_{\mu} \hat{D} \rightarrow-\kappa_{\mu}$ reduces to the Maxwell and Carroll-Field-Jackiw model [30]. However, it was shown in $[31,32]$ that this theory in time-like background does not produce superluminal velocities. So at this level one cannot expect to fit OPERA's data [1]. Thus, we should go to higher dimensional operators as in (1). Although such operators can produce superluminal velocities they comprise a Lorentz invariant Lagrangian except by the appearance of the Lorentz-violating background four vector $n^{\mu}[27,28]$, which can also be understood as a vacuum expectation value (VEV) of a vector field [24, 25]. For the sake of simplicity, we choose time-like backgrounds, which is not necessarily the frame of the neutrinos.

Let us now derive the dispersion relation associated to extended Myers-Pospelov dimension-5 operator in the fermionic sector. The equation of motion takes the form

$\left(i \not \partial-m-\tilde{g} \hat{D} \not h \gamma_{5}\right) \psi=0$.

After a straightforward algebra we find that the free continuous spectrum is governed by the following dispersion relation:

$\left(k^{2}-m^{2}-\tilde{g}^{2} n^{2} P_{n}^{2}\right)^{2}-4 \tilde{g}^{2} P_{n}^{3}=0$,

where

$P_{n}=(n \cdot k)^{2}-n^{2} k^{2}$.

For related issues in the original Myers-Pospelov model see, e.g., [33-35]—see also [36]. Hence, settling a timelike direction in the Lorentz-symmetry breaking background $n_{\mu}=\left(n_{0}, 0,0,0\right)$, we have 
$\left(\omega^{2}-|\boldsymbol{k}|^{2}-m^{2}-\tilde{g}^{2} n_{0}^{6}|\boldsymbol{k}|^{4}\right)^{2}-4 \tilde{g}^{2} n_{0}^{6}|\boldsymbol{k}|^{6}=0$

whose solutions are

$\omega_{ \pm}= \pm \sqrt{|\boldsymbol{k}|^{2}\left(1 \pm \tilde{g} n_{0}^{3}|\boldsymbol{k}|\right)^{2}+m^{2}}$.

We assume here the neutrino velocity is given by the group velocity determined from the fermionic dispersion relation (7), that is,

$v_{v}=\frac{\partial \omega_{+}}{\partial|\boldsymbol{k}|}=\frac{|\boldsymbol{k}|\left(1 \pm \tilde{g} n_{0}^{3}|\boldsymbol{k}|\right)\left(1 \pm 2 \tilde{g} n_{0}^{3}|\boldsymbol{k}|\right)}{\sqrt{|\boldsymbol{k}|^{2}\left(1 \pm \tilde{g} n_{0}^{3}|\boldsymbol{k}|\right)^{2}+m_{v}^{2}}}$.

Now expanding in large momenta $|\boldsymbol{k}|^{2} \gg m_{v}^{2}$, but keeping $\tilde{g} n_{0}^{3}|\boldsymbol{k}| \ll 1$ we find

$v_{v} \simeq 1 \pm 2 \tilde{g} n_{0}^{3}|\boldsymbol{k}|-\frac{m_{v}^{2}}{2|\boldsymbol{k}|^{2}}\left(1-4 \tilde{g}^{2} n_{0}^{6}|\boldsymbol{k}|^{2}\right)$.

If we keep only linear terms in $\tilde{g}$ we find (for the "plus sign" sector)

$v_{v}-1 \sim 2 \tilde{g} n_{0}^{3}|\boldsymbol{k}|-\frac{m_{v}^{2}}{2|\boldsymbol{k}|^{2}}$.

Note that for massless or almost massless fermions as in the case of neutrinos we have the possibility of superluminal velocities. We can indeed estimate the Lorentz violating coupling $\tilde{g}$ if we know the energy $E_{v} \sim|\boldsymbol{k}|$ and the mass $m_{v}$ of the superluminal neutrino satisfying the bound

$4 \tilde{g} n_{0}^{3}|\boldsymbol{k}|^{3}>m_{v}^{2}$

As we shall see below, this is easily satisfied by OPERA's data [1] where $\tilde{\alpha} \equiv \tilde{g} n_{0}^{3} \sim\left(1.7 \times 10^{6} \mathrm{GeV}\right)^{-1}$.

Let us now write the deviation of the neutrino velocity from the light speed (in the vacuum) as a function of momenta according to the curve with slope $\tilde{\alpha}$

$\frac{v_{v}-c}{c} \simeq 2 \tilde{\alpha}|\boldsymbol{k}|$,

where we are choosing $c=1$. For OPERA experiments $v_{v}-1 \sim 10^{-5}$ with $E_{v} \sim|\boldsymbol{k}| \sim 17 \mathrm{GeV}$ we find the slope

$\tilde{\alpha} \sim \frac{1}{1.7 \times 10^{6} \mathrm{GeV}} \sim 10^{-22} \mathrm{~m}$,

which sets our Lorentz-symmetry breaking parameter $\tilde{\alpha}$ to test superluminality up to the energy $1000 \mathrm{TeV}$, the energy scale present in primary cosmic rays originated in the explosion of massive stars [37]. Now substituting (13) into (12) we get the curve

$\frac{v_{v}-c}{c} \sim 10^{-5}\left(\frac{|\boldsymbol{k}|}{17 \mathrm{GeV}}\right)$.

Note this also agrees with MINOS experiment [38] with energy $E_{v} \sim|\boldsymbol{k}| \sim 3 \mathrm{GeV}$. To compare OPERA with supernova SN1987a measurements [39-41] we use $E_{v} \sim|\boldsymbol{k}| \sim 10$ $\mathrm{MeV}$ to obtain $\frac{v_{v}-c}{c} \sim 10^{-5}\left(\frac{10^{-3}}{1.7}\right)$,

which is consistent with the SN1987a bound $|v-c| / c<$ $2 \times 10^{-9}$ [39-41]. We conclude that the above constraints on the deviation from the light speed in superluminal neutrinos obey a linear curve fitting as a function of the energy with OPERA's slope $\tilde{\alpha} \sim 0.5 \times 10^{-6} \mathrm{GeV}^{-1}$. This has also been noticed in other recent investigations [42-44].

Similarly we can find the dispersion relation for the electromagnetic sector through its corresponding equation of motion

$\left(\partial^{2} \eta^{\mu \nu}-\partial^{\mu} \partial^{v}-2 g \hat{D} \varepsilon^{\nu \mu \lambda \rho} n_{\lambda} \partial_{\rho}\right) A_{v}=0$.

The photon dispersion relation in the time-like Lorentzviolating background is then given by

$\left(\omega^{2}-|\boldsymbol{k}|^{2}\right)^{2}-4 g^{2} n_{0}^{6}|\boldsymbol{k}|^{6}=0$.

Solving this equation we obtain the following solutions:

$\omega_{ \pm}(\boldsymbol{k})= \pm|\boldsymbol{k}| \sqrt{1 \pm 2 g n_{0}^{3}|\boldsymbol{k}|}$.

The group velocity here determines the photon velocity

$v_{\gamma}=\frac{\partial \omega_{+}}{\partial|\boldsymbol{k}|}=\frac{1 \pm 3 g n_{0}^{3}|\boldsymbol{k}|}{\sqrt{1 \pm 2 g n_{0}^{3}|\boldsymbol{k}|}}$.

For large momenta but keeping $\left|2 g n_{0}^{3} \boldsymbol{k}\right| \ll 1$ we find (for the "plus sign" sector)

$v_{\gamma}-1 \simeq 2 g n_{0}^{3}|\boldsymbol{k}|$.

Let us now use the group velocity for the photon given in (20) to write the deviation of the neutrino velocity from the light speed (in the presence of the Lorentz-violating background) as a function of momenta (up to linear terms)

$\frac{v_{v}-v_{\gamma}}{v_{\gamma}} \simeq 2(\tilde{\alpha}-\alpha)|\boldsymbol{k}|$.

Recall that $\tilde{\alpha} \equiv \tilde{g} n_{0}^{3}, \alpha \equiv g n_{0}^{3}, \tilde{g}=\eta / M$ and $g=\xi / M$, where we shall assume $n_{0}=1$ from now on. The expected superluminality of the neutrino (with mass $m_{v} \sim 0.2 \mathrm{eV}$ and energy $E_{v} \sim|\boldsymbol{k}| \sim 17 \mathrm{GeV}$ ) is negligible

$\frac{v_{\nu}-v_{\gamma}}{v_{\gamma}} \simeq 10^{-21}$.

By using (21) allows us to set a bound to the couplings $\eta$ and $\xi$ that measure the strength of the time-like Lorentzviolating background acting into the neutrino and photon sectors. Thus, at the Planck scale $M \sim 10^{19} \mathrm{GeV}$ it follows that

$\eta-\xi \simeq \frac{10^{-21} M}{2 \times 17 \mathrm{GeV}} \sim 2.5 \times 10^{-4}$.

The bound for $\eta$ can be found through the bound $\xi \sim 10^{-6}$ for photons as follows: 
$\frac{\eta}{\xi} \simeq 1+\frac{2.5 \times 10^{-4}}{\xi} \sim 10^{2}$,

which corresponds to $\eta \sim 10^{-4}$ for neutrinos. This means that at the Planck scale the neutrino interacts with the Lorentz violating background about 100 times as much as the photon interacts. Up to one order of magnitude these bounds approach those derived from renormalization group equations [27]—see also [45] for other bounds via dimension- 4 operators in purely space-like backgrounds.

If the couplings $\eta$ and $\xi$ change with energy keeping the difference $\eta-\xi$ then we consider it as the slope of the curve

$\delta v_{v}=\frac{v_{v}-v_{\gamma}}{v_{\gamma}} \simeq 2(\eta-\xi) \frac{|\boldsymbol{k}|}{M} \sim 5 \times 10^{-4} \frac{|\boldsymbol{k}|}{M}$.

It is interesting to note that if we bring the Planck scale to the $\mathrm{TeV}$ scale, i.e., $M=M_{\text {Earth }} \sim 1 \mathrm{TeV}$ and $|\boldsymbol{k}| \sim 17 \mathrm{GeV}$ one finds the OPERA's result, i.e., about $\delta v_{v} \sim 10^{-5}$, for the deviation of the neutrino velocity from the light speed. Furthermore, for the scale $M=M_{\text {Astro }}>1000 \mathrm{TeV}$ it is sufficient to avoid astrophysical constraints since one can readily find $\delta v_{v}<10^{-9}$. This is in accord with [25] since in the latter case the strength of the Lorentz-violating background $\tilde{\alpha}_{\text {Astro }} \sim 1 / M_{\text {Astro }}$ is about $10^{4}$ times smaller than $\tilde{\alpha}_{\text {Earth }} \sim 1 / M_{\text {Earth }}$ in the former case. One should note that the formula (25) can also be readily applied to electrons. Finally, noticed that for photons one makes use of (20) and $g \sim \tilde{g}(\xi \sim \eta)$ on the Earth—this is consistent with (23)— to find that $\delta v_{\gamma} \sim 10^{-5}$ at $|\boldsymbol{k}| \sim 17 \mathrm{GeV}$, a result also found in [25].

To bring the Planck scale to lower scales one should consider extra dimensions. In the five-dimensional RandallSundrum scenario [46] one finds $M_{5} \approx M_{\text {Planck }}$ for the warp factor $e^{k L}$ about $10^{15}$ and $M \equiv M_{5} e^{-k L} \sim 1 \mathrm{TeV}$ on the $\mathrm{TeV}$ (i.e., IR or Standard Model) brane.

Our Lagrangian is Lorentz invariant except by the appearance of the Lorentz-violating background four vector $n^{\mu}$. This is in the same class of the theories [24, 25, 27, 28]. As such, we can always choose a rest frame for the neutrino to study its decay to other particles. Because of the Lorentz invariance the energy-momentum is localy conserved and decay of highly energetic neutrinos into neutrinos and other particles with lower energies is forbidden [24]. This should be enough to evade the Cohen-Glashow bound [26]. Furthermore, we can fine tune $\xi$ and $\eta$ in order to make the Lorenz-violating background much stronger on the Earth than interstellar scale to avoid all the astrophysical constraints on Lorentz violation and Cohen-Glashow bound as well discussed in [25].

Acknowledgements We would like to thank CNPq, CAPES, PNPD/PROCAD-CAPES for partial financial support.

Open Access This article is distributed under the terms of the Creative Commons Attribution Noncommercial License which permits any noncommercial use, distribution, and reproduction in any medium, provided the original author(s) and source are credited.

\section{References}

1. T. Adam et al. (OPERA Collaboration), arXiv:1109.4897 [hepex]

2. S.S. Gubser, arXiv:1109.5687 [hep-th]

3. G.F. Giudice, S. Sibiryakov, A. Strumia, arXiv:1109.5682 [hep$\mathrm{ph}]$

4. J. Alexandre, arXiv:1109.5629 [hep-ph]

5. D. Autiero, P. Migliozzi, A. Russo, arXiv:1109.5378 [hep-ph]

6. F.R. Klinkhamer, arXiv:1109.5671 [hep-ph]

7. J. Ciborowski, J. Rembielinski, arXiv:1109.5599 [hep-ex]

8. G. Dvali, A. Vikman, arXiv:1109.5685 [hep-ph]

9. G. Amelino-Camelia, G. Gubitosi, N. Loret, F. Mercati, G. Rosati, P. Lipari, arXiv:1109.5172 [hep-ph]

10. A. Kehagias, arXiv:1109.6312 [hep-ph]

11. R.A. Konoplya, arXiv:1109.6215 [hep-th]

12. G. Cacciapaglia, A. Deandrea, L. Panizzi, arXiv:1109.4980 [hep$\mathrm{ph}]$

13. P. Wang, H. Wu, H. Yang, arXiv: 1110.0449 [hep-ph]

14. P. Wang, H. Wu, H. Yang, arXiv: 1109.6930 [hep-ph]

15. X.J. Bi, P.F. Yin, Z.H. Yu, Q. Yuan, arXiv:1109.6667 [hep-ph]

16. L. Iorio, arXiv:1109.6249 [gr-qc]

17. D. Fargion, D. D'Armiento, arXiv:1109.5368 [astro-ph.HE]

18. J. Franklin, arXiv:1110.0234v2 [physics.gen-ph]

19. L. Maccione, S. Liberati, D.M. Mattingly, arXiv:1110.0783 [hep$\mathrm{ph}]$

20. W. Winter, arXiv:1110.0424 [hep-ph]

21. E.N. Saridakis, arXiv: 1110.0697 [gr-qc]

22. J.S. Diaz, arXiv:1109.4620 [hep-ph]

23. F.R. Klinkhamer, G.E. Volovik, Pisma Zh. Eksp. Teor. Fiz. 94, 731 (2011). arXiv:1109.6624 [hep-ph]

24. S. Nojiri, S.D. Odintsov, Eur. Phys. J. C 71, 1801 (2011). arXiv: 1110.0889 [hep-ph]

25. T. Li, D.V. Nanopoulos, arXiv: 1110.3451 [hep-ph]

26. A.G. Cohen, S.L. Glashow, Phys. Rev. Lett. 107, 181803 (2011). arXiv: 1109.6562 [hep-ph]

27. R.C. Myers, M. Pospelov, Phys. Rev. Lett. 90, 211601 (2003). arXiv:hep-ph/0301124

28. D. Colladay, V.A. Kostelecky, Phys. Rev. D 55, 6760 (1997). arXiv:hep-ph/9703464

29. J. Alexandre, J. Ellis, N.E. Mavromatos, arXiv:1109.6296 [hep$\mathrm{ph}]$

30. S.M. Carroll, G.B. Field, R. Jackiw, Phys. Rev. D 41, 1231 (1990).

31. C. Adam, F.R. Klinkhamer, Phys. Lett. B 513, 245 (2001). arXiv:hep-th/0105037

32. J. Alfaro, A.A. Andrianov, M. Cambiaso, P. Giacconi, R. Soldati, Phys. Lett. B 639, 586 (2006). arXiv:hep-th/0604164

33. C.M. Reyes, L.F. Urrutia, J.D. Vergara, Phys. Rev. D 78, 125011 (2008). arXiv:0810.5379 [hep-ph]

34. C.M. Reyes, Phys. Rev. D 82, 125036 (2010). arXiv:1011.2971 [hep-ph]

35. T. Mariz, Phys. Rev. D 83, 045018 (2011). arXiv:1010.5013 [hepth]

36. S. Choubey, S.F. King, Phys. Lett. B 586, 353 (2004). arXiv: hep-ph/0311326

37. M. Amenomori et al. (Tibet AS(gamma) Collaboration), Phys. Rev. D 62, 112002 (2000). arXiv:astro-ph/0010292

38. P. Adamson et al. (MINOS Collaboration), Phys. Rev. D 76, 072005 (2007). arXiv:0706.0437 [hep-ex]

39. K. Hirata et al., Phys. Rev. Lett. 58, 1490 (1987)

40. R. Bionta, G. Blewitt, C. Bratton, D. Casper, A. Ciocio et al., Phys. Rev. Lett. 58, 1494 (1987)

41. M.J. Longo, Phys. Rev. D 36, 3276 (1987) 
42. M.A. Anacleto, F.A. Brito, E. Passos, arXiv:1109.6298 [hep-th]

43. F. Tamburini, M. Laveder, arXiv: 1109.5445 [hep-ph]

44. J. Ellis, H.T. Janka, N.E. Mavromatos, A.S. Sakharov, E.K.G. Sarkisyan, arXiv:1110.4848 [hep-ph]
45. R. Casana, M.M. Ferreira Jr., J.S. Rodrigues, Phys. Rev. D 78, 125013 (2008). arXiv:0810.0306 [hep-th]

46. L. Randall, R. Sundrum, Phys. Rev. Lett. 83, 3370 (1999). arXiv: hep-ph/9905221 